\title{
Cavity quantum electrodynamics in the Anderson-localized regime
}

Sapienza, Luca; Nielsen, Henri Thyrrestrup; Stobbe, Søren; Garcia-Fernández, David; Smolka, Stephan; Lodahl, Peter

Published in:

2010 Conference on Lasers and Electro-Optics (CLEO) and Quantum Electronics and Laser Science

Conference (QELS)

Publication date:

2010

Document Version

Publisher's PDF, also known as Version of record

Link back to DTU Orbit

Citation (APA):

Sapienza, L., Nielsen, H. T., Stobbe, S., Garcia-Fernández, D., Smolka, S., \& Lodahl, P. (2010). Cavity quantum electrodynamics in the Anderson-localized regime. In 2010 Conference on Lasers and Electro-Optics (CLEO) and Quantum Electronics and Laser Science Conference (QELS) (pp. 1-2). IEEE.

\section{General rights}

Copyright and moral rights for the publications made accessible in the public portal are retained by the authors and/or other copyright owners and it is a condition of accessing publications that users recognise and abide by the legal requirements associated with these rights.

- Users may download and print one copy of any publication from the public portal for the purpose of private study or research.

- You may not further distribute the material or use it for any profit-making activity or commercial gain

- You may freely distribute the URL identifying the publication in the public portal 


\title{
Cavity Quantum Electrodynamics in the Anderson- localized Regime
}

\author{
Luca Sapienza, Henri Thyrrestrup, Søren Stobbe, Pedro David Garcia, Stephan Smolka, and Peter \\ Lodahl \\ DTU Fotonik, Department of Photonics Engineering, Technical University of Denmark, Ørsteds Plads 343, DK-2800 Kgs. Lyngby, \\ Denmark. www.fotonik.dtu.dk/quantumphotonics \\ Authore-mail address: lucs@fotonik.dtu.dk
}

\begin{abstract}
We experimentally measure, by means of time-resolved photoluminescence spectroscopy, a 15-fold enhancement of the spontaneous emission decay rate of single semiconductor quantum dots coupled to disorder-induced Anderson-localized modes with efficiencies reaching $94 \%$.

(C2010 Optical Society of America

OCIS codes: (270.5580) Quantum electrodynamics; (130.5296) Photonic crystal waveguides
\end{abstract}

Enhancing the spontaneous emission decay rate of single quantum emitters is crucial in the realisation of efficient single-photon sources, necessary for the development of quantum information technology and quantum cryptography. The control over the emission dynamics of atoms, ions or semiconductor quantum dots is typically achieved by placing the quantum emitters within highly confining optical cavities [1]. The modified density of photonic states produces an increase of the spontaneous emission decay rates, through the so-called Purcell factor, and the enhanced light-matter interaction can even allow to entangle single photons and single emitters.

We demonstrate a new way to enhanced light-matter interaction based on the control over the disorder of the system enabling quantum electrodynamics experiments [2]. By engineering the degree of disorder in the system, light can be localized by multiple scattering events and be trapped within so-called Andersonlocalized cavities [3]. To this end, we have realised a sample composed of a photonic crystal waveguide (W1 with lattice parameter $a=260 \mathrm{~nm}$, hole radius $r=78 \mathrm{~nm}$ ) containing a layer of self assembled InAs $/ \mathrm{GaAs}$ quantum dots in the centre. Disorder is introduced in the photonic crystal membrane by randomly shifting the position of the air holes, with respect to the perfectly periodic structure, in three rows on either side of the waveguide (see Fig.1A).

A

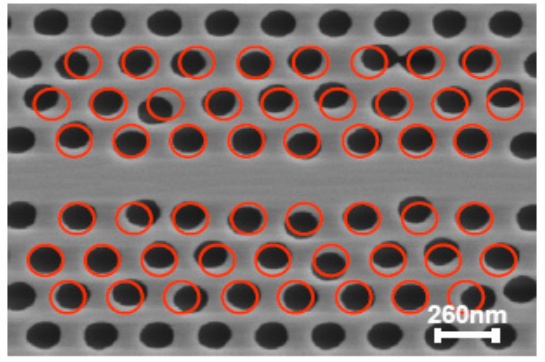

B

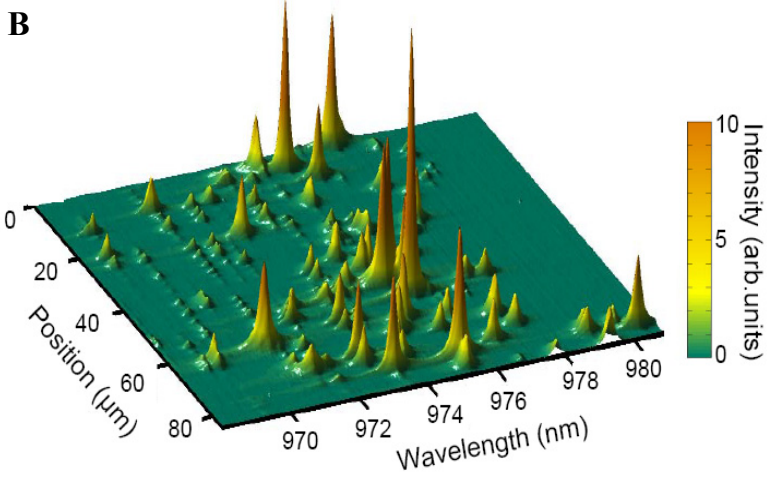

Fig. 1: (A) Scanning electron micrograph of a GaAs photonic crystal waveguide with engineered disorder in the position of the air holes. The red circles represent the hole positions in an ideal structure without induced disorder. (B) High-power photoluminescence spectra collected while scanning the excitation and collection microscope objective along the waveguide (sample temperature: $10 \mathrm{~K}$ ). 


\section{QPDA7.pdf}

The induced disorder randomizes the propagation of light along the waveguide and Anderson-localized cavities appear spontaneously. Sharp peaks are visible in high power photoluminescence spectra (see Fig.1B) that are spectral signatures of Anderson-localized cavities. The frequency range where the cavities appear can be controlled by varying the amount of disorder and lies within the waveguide slow-light regime. Remarkably, the measured $Q$-factors (up to 10000) and mode volumes $\left(\sim 1 \mu \mathrm{m}^{3}\right)$ compete with highly engineered semiconductor cavities containing quantum dots [4].

The modification of the spontaneous emission rate of single quantum dots embedded within disordered photonic crystal waveguides is studied by means of time-resolved photoluminescence spectroscopy. The emission frequency of single semiconductor quantum dots is tuned into and out of resonance with the random cavity modes by varying the temperature of the sample. The decay rate of single emitters proves to be strongly influenced by the modified local density of photonic states. A 15-fold enhancement of the spontaneous emission rate is measured when tuning a single quantum dot on and off resonance with a random cavity (see Fig.2). The measured efficiency in the channelling of single photons into an Andersonlocalized mode reaches values as high as $94 \%$. These results prove that disorder induced Andersonlocalized cavities represent a novel and efficient platform for all-solid-state quantum electrodynamics. The implementation of disorder represents a novel route to the enhancement of light-matter interaction, opening exciting perspectives both in fundamental research and in the realization of intrinsically "imperfectionrobust" quantum information devices. So-called necklace states that are naturally occurring coupled Anderson-localized modes, could also offer a novel route to the realization of quantum networks [5].

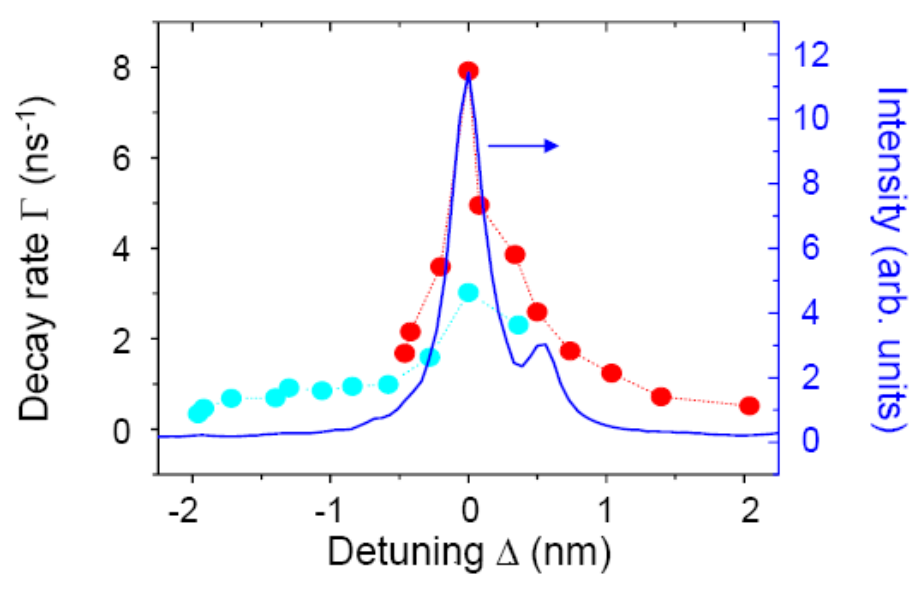

Fig. 2: Decay rates of two different quantum dots (red and blue filled circles) coupled to an Anderson-localized cavity, plotted as a function of the emission wavelength detuning with respect to the cavity. The cavity emission spectrum is plotted in the solid line.

[1] K. J. Vahala, “Optical microcavities”, Nature 424, 839 (2003).

[2] L.Sapienza, H. Thyrrestrup, S. Stobbe, P. D. Garcia, S. Smolka, and P. Lodahl, "Cavity Quantum Electrodynamics with Anderson-Localized Modes", Science 327, 1352 (2010).

[3] J. Topolancik, B. Ilic, F. Vollmer, "Experimental Observation of Strong Photon Localization in Disordered Photonic Crystal Waveguides", Phys. Rev. Lett. 99, 253901 (2007).

[4] K. Hennessy et al., “Quantum nature of a strongly coupled single quantum dot-cavity system”, Nature 445, 896 (2007).

[5] D.S. Wiersma, "Random Quantum Networks “, Science 327, 1333 (2010). 